\title{
MULTIDRUG-RESISTANT POLYMICROBIAL BACTEREMIA AND SEPSIS: A RARE CASE REPORT
}

\author{
SIAVASH SHAHBAZI NIA ${ }^{1 *}$, SHADAN MODARESAHMADI ${ }^{1}$, VITHYA THIRUMOORTHI ${ }^{1}$, QAISAR ANWAR $^{2}$, \\ SHANKAR PRASAD ${ }^{2}$
}

${ }^{1}$ Department of Pharmacy Practice, Al-Ameen College of Pharmacy, Bengaluru, Karnataka, India. ${ }^{2}$ Department of General Medicine, St. Philomena's Hospital, Bengaluru, Karnataka, India. Email: siavashshahbazinia6640@gmail.com

Received: 27 April 2018, Revised and Accepted: 24 May 2018

ABSTRACT

Antibiotic-resistance is currently a serious threatening factor to global public health that needs a universal action by all government sectors and societies. Here, we report an aggressive case of multidrug-resistant (MDR) polymicrobial bacteremia and sepsis. A 73-year-old male patient was admitted to a tertiary hospital with the complaints of breathlessness, cough with expectorations, vomiting, loose stools, and altered sensorium due to polymicrobial bacteremia and sepsis, which was found to be antibiotic resistant. Blood culture reports showed Pseudomonas aeruginosa and Pseudomonas putida, and sputum culture showed Acinetobacter baumannii and Klebsiella pneumonia. However, according to the culture sensitivity test reports, the organisms were resistant to almost all antibiotics and different antibiotics were tried. On the last day of hospitalization, the patient started desaturation and developed bradycardia and after sometimes cardiac arrest. In spite of continuous cardiopulmonary resuscitation, the patient could not be resuscitated and declared to be dead. The patient was finally diagnosed with MDR polymicrobial bacteremia and sepsis, chronic obstructive pulmonary disease with cor pulmonale, colitis, diabetes mellitus, hypertension, and respiratory acidosis. Although polymicrobial bacteremia and sepsis are rare, awareness of such conditions and of the rare causative pathogens, such as A. baumannii, especially in patients with comorbidities, will help treat the patients with bacteremia and sepsis.

Key words: Antibiotic resistance, Tertiary hospital, Sepsis.

(C) 2018 The Authors. Published by Innovare Academic Sciences Pvt Ltd. This is an open access article under the CC BY license (http://creativecommons. org/licenses/by/4. 0/) DOI: http://dx.doi.org/10.22159/ajpcr.2018.v11i9.26965

\section{INTRODUCTION}

Antimicrobial resistance is a modern warfare against the numerous numbers of microorganisms, which are constantly evolving [1]. In the last decade, a remarkable increase has been observed in proportion as well as in the number of bacterial pathogens exhibiting resistance to antibacterial agents. The United States Centers for Disease Control and Prevention (USCDC) and the World Health Organization consider the infection caused by resistant bacteria as an emergent global disease and a major public health problem [2]. From the time when sulfonamide and penicillin were introduced into clinical use in the 1930s and 1940s, respectively, people were captured by the illusion that all infectious diseases could be totally controlled by antibiotics [3]. This was while the prolonged and repeated courses of antibiotics exposure were compelling the development of antibiotic-resistant organisms [4].

Universal, rapid appearance of resistant bacteria endangers the efficacy of antibiotics, which have considerably saved millions of lives. In 1945, Sir Alexander Fleming raised the alarm regarding antibiotic overuse, which clearly drives the evolution of resistance [5]. Infection with antibiotic-resistant bacteria may lead to severe illness, increased mortality rates, and an increased risk of complications and admission to hospital [6]. Failure to apply infection control measures in a hospital and outside it can be an important cause for the spread of antibiotic resistance [7]. Infections caused by multidrug-resistant (MDR) organisms can cause consequential economic burden, estimated to be more than 20 billion dollars per year in the United States of America (USA) only. The USCDC reports that more than 23,000 people in the USA are dying per year due to an infection with an antibiotic-resistant organism. Moreover, it anticipates that antibiotic resistance can cause 300 million premature deaths by 2050 , with a global economic loss up to $\$ 100$ trillion [8]. Antibiotic resistance is a global concern since new resistance mechanisms are appearing and spreading globally, minimizing our ability to treat common infectious diseases, thereby leading to prolonged illness, disability, and death [9]. Hence, hereby, we report a 73-year-old male patient with the complaints of breathlessness, cough with expectorations, vomiting, loose stools, and altered sensorium due to rare condition of MDR polymicrobial bacteremia and sepsis caused by rare pathogens, such as Acinetobacter baumannii so as to make an awareness regarding jeopardy of antibiotic-resistance and its consequences $[10,11]$.

\section{CASE REPORT}

A 73-year-old male patient was admitted to a tertiary care hospital with the presenting complaints of breathlessness, cough with expectorations, vomiting, loose stools, and altered sensorium. Hence, he was shifted to the Intensive Care Unit. The patient also developed abdominal distention and did not pass stools since the time of admission.

The patient had a history of chronic obstructive pulmonary disease (COPD) with cor pulmonale, diabetes mellitus, and hypertension. He was admitted to the hospital, twice earlier for exacerbation of COPD and bilateral lower lobe pneumonia. His first admission was in January 2016, and he was hospitalized for a period of 6 days. His second admission was on August 2016, and he was hospitalized for 8 days.

According to the patient's history, he was apparently fine until 3 days before the admission when he developed breathlessness and cough with expectorations, which was yellowish in color, vomiting 3-4 episodes, and loose stools $4-5$ times.

On admission (December 2016), blood pressure (BP) was found to be $120 / 80 \mathrm{mmHg}$, pulse rate was $102 \mathrm{bpm}$, general random blood sugar was $110 \mathrm{mg} / \mathrm{dl}$, respiratory rate was $22 \mathrm{bpm}, \mathrm{O}_{2}$ saturation was $86 \%$ in room air, and temperature was $98.6^{\circ} \mathrm{F}$. Complete blood count results showed elevated white blood cell (WBC) and neutrophil level. The patient was prescribed with intravenous amoxicillin-clavulanate thrice a day, tablet amlodipine twice a day, tablet rosuvastatin once a day, tablet sildenafil twice a day, nebulizer ipratropium bromide-salbutamol thrice a day, and nebulizer budesonide thrice a day. Insulin therapy was also started for the patient to control his blood glucose level. 
On day 2, the patient became drowsy, and he was not obeying commands. Arterial blood gas report showed elevated $\mathrm{pCo}_{2}$. The chest X-ray results showed bilateral lower lobe haziness. As the patient's WBC count has increased, the physician considered the previous antibiotic to be ineffective and stopped it. Simultaneously, he started intravenous levofloxacin once a day and continued the remaining treatment. In addition, the blood sample was sent for susceptibility analysis.

On day 3 , the patient still had cough with expectoration and breathlessness. He developed abdominal distension, and his BP was raised to $140 / 90 \mathrm{mmHg}$. Electrocardiography also showed sinus tachycardia. The physician added intravenous cefoperazone-sulbactam twice a day, intravenous metronidazole thrice a day, and intravenous pantoprazole once a day and continued the remaining medicines.

On day 4 , the patient became drowsier, and his BP was raised to $170 / 110 \mathrm{mmHg}$. He was febrile, and WBC count was almost same. The physician substituted intravenous cefoperazone-sulbactam with intravenous meropenem thrice a day. The patient was also prescribed with intravenous enoxaparin twice a day and intravenous paracetamol thrice a day, infusion fentanyl and remaining medicines were continued. Moreover, the patient had been put on ventilator support and undergone exploratory laparoscopy due to his abdominal distention. From the exploratory laparoscopy, it was found that a segment of jejunum about $15 \mathrm{~cm}$ in length, starting $10 \mathrm{~cm}$ from the duodenal-jejunal junction, was congested and thickened. Moreover, exploratory laparoscopy showed good peristaltic activity, and there was no evidence of necrotic patches.

On day 5, 6, 7, and 8 of hospitalization, the patient had fever spike $\left(105^{\circ}\right.$ F), and WBC count had reduced. However, on day 6 of hospitalization, blood culture reports showed Pseudomonas putida to be present. Susceptibility information for P. putida is presented in Table 1.

On day 9 , the patient was trying to open his eyes, and he was responding to verbal commands. The physician advised physiotherapy.

On day 10, still, the patient was responding to verbal commands. His WBC count had drastically elevated up to 40000 cells/cumm. In addition, the neutrophil level also increased to $91 \%$. Hence, the physician stopped intravenous levofloxacin and added intravenous fluconazole once a day and intravenous clindamycin thrice a day. In addition, blood and sputum samples were sent for susceptibility analysis.

On day 11, still, the patient had fever spikes. The patient was given intravenous amikacin twice a day.

On day 12,13 , and 14 , the patient opened his eyes to commands. The patient was febrile and his BP was normal. WBC count, as well as neutrophil level, was still high. On day 14 of hospitalization, blood culture reports showed Pseudomonas aeruginosa, and sputum culture showed A. baumannii to be present. Same medication had been continued during these 3 days. On Table 2, susceptibility information for P. aeruginosa, and on Table 3, susceptibility information for A. baumannii can be observed.

On day 15, the patient's BP was 150/80 mmHg. He was still febrile. His WBC count and neutrophil level have decreased. However, sputum culture showed Klebsiella pneumonia to be present. The physician stopped intravenous meropenem and started intravenous doxycycline twice a day and intravenous colistin twice a day. Table 4 represents the susceptibility information for K. pneumonia.

On day 16, the patient had elevated BP. The physician stopped intravenous amikacin and intravenous fluconazole.

On day 17, the patient was febrile, conscious and he had nasal bleeding. Ear-nose-throat (ENT) specialist opinion sought and tracheostomy was done since the patient was on prolonged ventilation.
On day 18, abdominal distension was still present, and the patient developed bilateral pedal edema. His WBC count started to increase. Intravenous colistin and intravenous clindamycin were stopped, and intravenous tigecycline twice a day was started.

On day 19 , the patient had a very poor general condition. His BP was $90 / 40 \mathrm{mmHg}$. Despite the patient had received different antibiotics, his condition did not change. Hence, the physician shifted the patient to the isolation room. The patient started desaturation and developed bradycardia. Hence, 1 ampule atropine was given, but the patient developed cardiac arrest. In spite of continuous cardiopulmonary resuscitation and administering adrenaline, the patient could not be resuscitated and declared to be dead.

According to the susceptibility analysis reports presented in Tables 1-4, different antibiotics were tried. In addition to antibiotics, antifungal, diuretic, heparin, parenteral nutrition, analgesic, antacid, and other supportive measures were used during his stay of 19 days in the hospital. His final diagnosis was MDR polymicrobial bacteremia and sepsis, COPD with cor pulmonale, lower respiratory tract infection, colitis, diabetes mellitus, and hypertension.

\section{DISCUSSION}

Antibiotic resistance is one of the biggest threats to global health, which can affect anyone, of any age, in any country. Antibiotic resistance occurs naturally, but misuse of antibiotics in humans and animals is accelerating the process [10].

In the aforementioned case, we are observing a rare condition of MDR polymicrobial bacteremia and sepsis, which had been caused by multiple pathogens, namely, P. aeruginosa, P. putida, A. baumannii, and $K$. pneumonia among which $A$. baumannii is a rare causative organism[10,11].

In this case, rational use of antibiotics can be observed to some extent. In other words, only a few numbers of antibiotics were prescribed or substituted with other agents based on culture reports. However, the majority of changes in antibiotic therapy were based on changes in WBC and neutrophil counts or persistence of fever in the patient. In the $1^{\text {st }}$ day of admission, as past history of the patient was not fully known, based on the general rule for the treatment of infectious diseases, a broad-spectrum antibiotic (amoxicillin-clavulanate) had been prescribed to the patient until the exact pathogens were known [12]. After the patient's history was understood, as he had two previous admissions for pneumonia and COPD, amoxicillin-clavulanate was considered to be ineffective and therefore substituted with levofloxacin. In addition, the increment in WBC count was also influential in taking decision for this change of antibiotic. On the $3^{\text {rd }}$ day of admission, cefoperazone-sulbactam and metronidazole were added to the patient's prescription. However, on the $4^{\text {th }}$ day of admission, due to the high and constant value of WBC count, cefoperazone-sulbactam had been substituted with meropenem as it is a broad-spectrum antibiotic and covers the majority of gram-negative and gram-positive organisms. On day 6 of hospitalization, blood culture reports showed P. putida (Table 1) to be present, and it was found that P. putida was resistant to the majority of antibiotics. As on the $10^{\text {th }}$ day of admission, the patient's condition had been worsened, and the physician stopped levofloxacin. As in the culture report, the sensitivity of $P$. putida to clindamycin had not been checked, and there were chances of sensitiveness of $P$. putida to this antibiotic. Hence, physician started this antibiotic and also added fluconazole for prophylactic purposes. On the $11^{\text {th }}$ day of hospitalization, still the patient had fever spike, and his condition did not improve. Hence, he was suspected to be infected by some other organisms. As a result, amikacin was started empirically. On the $14^{\text {th }}$ day of hospitalization, WBC count, as well as neutrophil level, was still high. Blood culture reports showed P. aeruginosa (Table 2) and sputum culture showed A. baumannii (Table 3 ) to be present and both were found to be sensitive to colistin. Hence, colistin was started. On the next day, from sputum culture, it was found that K. pneumonia 
Table 1: Susceptibility information for Pseudomonas putida performed on day 6

\begin{tabular}{llll}
\hline Antibiotic & Interpretation & Antibiotic & Interpretation \\
\hline Amoxicillin/Clavulanate & $\mathrm{R}$ & Ciprofloxacin & Tigecycline \\
Ampicillin & $\mathrm{R}$ & Nitrofurantoin & $\mathrm{R}$ \\
Ceftriaxone & $\mathrm{R}$ & Nalidixic acid & $\mathrm{R}$ \\
Cefuroxime & $\mathrm{R}$ & Meropenem & $\mathrm{R}$ \\
Cefuroxime axetil & $\mathrm{R}$ & Imipenem & $\mathrm{R}$ \\
Cefepime & $\mathrm{R}$ & Ertapenem & $\mathrm{R}$ \\
Cefoperazone/Sulbactam & $\mathrm{R}$ & Colistin & $\mathrm{S}$ \\
Piperacillin/Tazobactam & $\mathrm{R}$ & Trimethoprim/Sulfamethoxazole \\
Gentamicin & $\mathrm{R}$ & - & $\mathrm{R}$ \\
Amikacin & $\mathrm{R}$ & - & - \\
Levofloxacin & $\mathrm{R}$ & & \\
\hline
\end{tabular}

S: Sensitive, R: Resistant

Table 2: Susceptibility information for Pseudomonas aeruginosa performed on day 14

\begin{tabular}{llll}
\hline Antibiotic & Interpretation & Antibiotic & Interpretation \\
\hline Ceftazidime & $\mathrm{R}$ & Minocycline & $\mathrm{R}$ \\
Cefepime & $\mathrm{R}$ & Tigecycline & $\mathrm{R}$ \\
Cefoperazone/Sulbactam & $\mathrm{R}$ & Meropenem & $\mathrm{R}$ \\
Piperacillin/Tazobactam & $\mathrm{R}$ & Doripenem & $\mathrm{R}$ \\
Ticarcillin/Clavulanate & $\mathrm{R}$ & Imipenem & $\mathrm{R}$ \\
Gentamicin & $\mathrm{R}$ & Aztreonam & $\mathrm{R}$ \\
Amikacin & $\mathrm{R}$ & Colistin & $\mathrm{S}$ \\
Ciprofloxacin & $\mathrm{R}$ & Trimethoprim/Sulfamethoxazole \\
Levofloxacin & $\mathrm{R}$ & - & $\mathrm{R}$ \\
\hline
\end{tabular}

S: Sensitive, R: Resistant

Table 3: Susceptibility information for Acinetobacter baumannii performed on day 14

\begin{tabular}{llll}
\hline Antibiotic & Interpretation & Antibiotic & Interpretation \\
\hline Ceftazidime & $\mathrm{R}$ & Minocycline & $\mathrm{R}$ \\
Cefepime & $\mathrm{R}$ & Tigecycline & Meropenem \\
Cefoperazone/Sulbactam & $\mathrm{S}$ & Doripenem & $\mathrm{R}$ \\
Piperacillin/Tazobactam & $\mathrm{R}$ & $\mathrm{R}$ \\
Ticarcillin/Clavulanate & - & Imipenem & Aztreonam \\
Gentamicin & $\mathrm{R}$ & Colistin & $\mathrm{R}$ \\
Amikacin & - & Trimethoprim/Sulfamethoxazole \\
Ciprofloxacin & $\mathrm{R}$ & $\mathrm{S}$ \\
Levofloxacin & $\mathrm{R}$ & - & $\mathrm{R}$ \\
\hline
\end{tabular}

S: Sensitive, R: Resistant

Table 4: Susceptibility information for Klebsiella pneumonia performed on day 15

\begin{tabular}{llll}
\hline Antibiotic & Interpretation & Antibiotic \\
\hline Amoxicillin & $\mathrm{R}$ & Amikacin & $\mathrm{R}$ \\
Amoxicillin/clavulanate & $\mathrm{R}$ & Ciprofloxacin & $\mathrm{R}$ \\
Ampicillin & $\mathrm{R}$ & Tigecycline & Nitrofurantoin \\
Ceftriaxone & $\mathrm{R}$ & Nalidixic acid & $\mathrm{R}$ \\
Cefotaxime & $\mathrm{R}$ & Meropenem \\
Cefuroxime & $\mathrm{R}$ & Imipenem \\
Cefuroxime axetil & $\mathrm{R}$ & Ertapenem \\
Cefepime & $\mathrm{R}$ & Colistin \\
Cefoperazone/Sulbactam & $\mathrm{R}$ & $\mathrm{R}$ \\
Piperacillin/Tazobactam & $\mathrm{R}$ & Trimethoprim/Sulfamethoxazole \\
Gentamicin & $\mathrm{R}$ & - & $\mathrm{R}$ \\
\hline
\end{tabular}

R: Resistant, I: Intermediate

(Table 4) sensitive to tetracyclines was present. As a result, doxycycline was prescribed to the patient. On the $18^{\text {th }}$ day of admission, since the patient's condition started worsening, tigecycline was also prescribed to get benefit from its synergistic effect with doxycycline. However, unfortunately, none of these many antibiotics were effective against the microorganisms infected the patient.

In general, management of antibiotic resistance is an attempt to slow the spread of resistance through prudent use of antibiotics based on guidelines of antimicrobial stewardship programs and various data such as pharmacokinetic and pharmacodynamic properties of antibiotics, diagnostic testing, antimicrobial susceptibility testing, clinical response, and effects on the microbiota, as well as by new antibiotic developments. Evolution of antibiotic resistance can be slowed by minimizing the antibiotic use and avoidance from treating asymptomatic infections. Antibiotics must be used only if they are considered to be effective and when treatment is essential as little antibiotic as possible must be used. Moreover, through use of vaccines, 
hygiene, and isolation of infected patients, the need for antibiotic treatment can be minimized $[13,14]$.

\section{FUNDING}

Nil.

\section{CONFLICT OF INTEREST}

The authors declare that there is no conflict of interest regarding the publication of this paper.

\section{AUTHOR'S CONTRIBUTION}

Dr. Siavash Shahbazi Nia and Dr. Shadan Modaresahmadi actively participated in data collection and preparation of the manuscript. Dr. Vithya Thirumoorthi did the primary correction and Dr. Qaisar Anwar, who was involved in the treatment of the patient, did the final correction of the manuscript. Dr. Shankar Prasad approved the final version of the manuscript.

\section{REFERENCES}

1. Divyashanthi CM, Adithiyakumar S, Bharathi N. Study of prevalence and antimicrobial susceptibility pattern of bacterial isolates in a tertiary care hospital. Int J Pharm Pharm Sci 2015;7:185-90.

2. Roca I, Akova M, Baquero F, Carlet J, Cavaleri M, Coenen S, et al. The global threat of antimicrobial resistance: Science for intervention. New Microbes New Infect 2015;6:22-9.

3. Yoneyama H, Katsumata R. Antibiotic resistance in bacteria and its future for novel antibiotic development. Biosci Biotechnol Biochem
2006;70:1060-75

4. Shinde AR, Mohite RV, Shinde RV. Pattern of antibiotics utilization in neonatal septicemia: A cross-sectional study from rural tertiary care hospital Western Maharashtra, India. Int J Pharm Pharm Sci 2017;9:60-3

5. Ventola CL. The antibiotic resistance crisis. P T 2015;40:277-83.

6. Lior C, Bjerrum L. Antimicrobial resistance: Risk associated with antibiotic overuse and initiatives to reduce the problem. Ther Adv Drug Saf 2014;5:229-41.

7. Giedraitiene A, Vitkauskiene A, Naginiene R, Pavilonis A. Antibiotic resistance mechanisms of clinically important bacteria. Medicina (Kaunas) 2011;47:137-46

8. Munita JM, Arias CA. Mechanisms of antibiotic resistance. Microbiol Spectr 2016;4:doi: 10.1128/microbiolspec.

9. World Health Organization. Antibiotic Resistance; 2017 Available from: http://www.who.int/mediacentre/factsheets/antibiotic-resistance/ en/. [Last accessed on 2017 Oct 02].

10. Arahata K, Yamaguchi R, Terashima T. A case of polymicrobial bacteremia in a patient undergoing chemotherapy. Hindawi 2018;2018:1-4

11. Son YW, Jung IY, Ahn MY, Jeon YD, Ann HW, Ahn JY, et al. A case of community-acquired pneumonia caused by multidrugresistant Acinetobacter baumannii in Korea. Infect Chemother 2017;49:297-300.

12. National Health Service. Sepsis-Treatment; 2016. Available from http:// www.nhs.uk/Conditions/Blood-poisoning/Pages/Treatment.aspx. [Last Accessed on 2017 Oct 02].

13. Read AF, Woods RJ. Antibiotic resistance management. Evol Med Public Health 2014;2014:147.

14. Lee CR, Cho IH, Jeong BC, Lee SH. Strategies to minimize antibiotic resistance. Int J Environ Res Public Health 2013;10:4274-305. 